\title{
Density Functionals for the Yukawa Electron-Electron Interaction
}

\author{
ANDREAS SAVIN \\ Laboratoire Dynamique des Interaction Moléculaires, Université Pierre et Marie Curie, 4, place \\ Jussieu, F-75252 Paris, France \\ HEINZ-JÜRGEN FLAD \\ Institut für Theoretische Chemie, Universität Stuttgart, Pfaffenwaldring 55, D-70569 Stuttgart, \\ Germany
}

Received June 13, 1994; accepted September 28, 1994

\begin{abstract}
Short-range nonclassical electron-electron interaction is described by a density functional in a scheme that allows multideterminant wave functions. The parameter that determines the coupling with the configuration-interaction-type calculations can be chosen in a controlled manner. Results are presented for the He and the Be series using a Yukawa-type interaction. (c) 1995 John Wiley \& Sons, Inc.
\end{abstract}

\section{Introduction}

A Ithough improving the density functionals as defined by Kohn and co-workers $[1,2]$ is certainly a very successful approach, one may alternatively consider the possibility of extending their theory to exploit the experience gained in wave-function calculations using multideterminant wave functions. The difficulty comes mainly from the problem of finding a proper separation between the contributions coming from the density functional and that which is due to the wave function. It seems physically sound to try to describe the short-range interaction of electrons by density functionals (e.g., the cusp condition) and to use wave functions for the long-range part (cf. the hydrogen molecule at large internuclear distances). This philosophy is also supported by practical point of view: It is known that short-range interaction leads to a slowly convergent expansion of the wave function in terms of Slater determinants (see, e.g., [3]), whereas near-degeneracy effects can be well described with medium-sized basis sets [4]).

There are several other approaches that relate configuration-interaction-type calculations with density-functionals [5-13]. A comparison of different approaches can be found in [12]. 


\section{Theory}

The existence of universal short-range densityfunctionals is most easily shown in the constrainedsearch formalism ([14], see also, [15]). Let $\hat{T}$ be the operator for kinetic energy, and $\hat{V}_{e e}$, that for the electron-electron interaction. Let further $\Psi_{n}^{\min }$ be the antisymmetric wave function that minimizes $\left\langle\hat{T}+\hat{V}_{e e}\right\rangle$ and yields the density $n$. Then, according to Levy [14], the universal density functional needed for obtaining the ground-state energy is

$$
F[n]=\left\langle\Psi_{n}^{\min }\left|\hat{T}+\hat{V}_{e e}\right| \Psi_{n}^{m i n}\right\rangle .
$$

With the classical electron-electron interaction,

$$
U[n]=\frac{1}{2} \int n\left(\mathbf{r}_{1}\right) n\left(\mathbf{r}_{2}\right) \frac{1}{r_{12}} d^{3} r_{1} d^{3} r_{2},
$$

and with $\Phi_{n}^{\min }$, the single determinant that yields $n$ and minimizes $\langle\hat{T}\rangle$, the universal density functionals for exchange-correlation

$$
E_{x c}[n]=F[n]-\left(\left\langle\Phi_{n}^{\min }|\hat{T}| \Phi_{n}^{\min }\right\rangle+U[n]\right)
$$

and correlation

$$
E_{c}[n]=F[n]-\left(\left\langle\Phi_{n}^{m i n}|\hat{T}| \Phi_{n}^{m i n}\right\rangle+\left\langle\Phi_{n}^{m i n}\left|\hat{V}_{e e}\right| \Phi_{n}^{m i n}\right\rangle\right)
$$

can be defined.

The last two formulas can be modified to define universal short-range functionals. One can split $\hat{V}_{e e}$ into a short-range $\left(\hat{V}_{s}\right)$ and a long-range $\left(\hat{V}_{l}\right)$ part (cf. [8]):

$$
\hat{V}_{e e}=\hat{V}_{s}+\hat{V}_{l} .
$$

For example, $\hat{V}_{s}$ can be chosen of the Yukawa type:

$$
\begin{aligned}
\hat{V}_{s} & =\sum_{i<j} v_{s}(i, j) \\
v_{s}(i, j) & =\frac{e^{-\mu r_{i j}}}{r_{i j}} .
\end{aligned}
$$

One then can define as universal density functionals for short-range exchange-correlation

$E_{s, x c}[n]=F[n]-\left(\left\langle\Phi_{n}^{m i n, l}\left|\hat{T}+\hat{V}_{l}\right| \Phi_{n}^{\text {min,l }}\right\rangle+U_{s}[n]\right)$, or for short-range correlation only:

$$
\begin{aligned}
E_{s, c}[n]=F[n]- & \left(\left\langle\Phi_{n}^{\min , l}\left|\hat{T}+\hat{V}_{l}\right| \Phi_{n}^{m i n, l}\right\rangle\right. \\
& \left.\left.+\Phi_{n}^{\min }\left|\hat{V}_{s}\right| \Phi_{n}^{\min }\right\rangle\right) .
\end{aligned}
$$

These formulas have been obtained by replacing the operator $\hat{T}$ by the operator $\hat{T}+\hat{V}_{l}$. Here, $\Phi_{n}^{\min , l}$ is the antisymmetric wave function that yields $n$ and minimizes $\left\langle\hat{T}+\hat{V}_{l}\right\rangle$. By this choice, it is possible to introduce a multideterminant wave function $\left(\Phi_{n}^{\min , l}\right)$ instead of a single-determinant one $\left(\Phi_{n}^{\min }\right)$. With a suitable choice of $\hat{V}_{l}$ [as with the Yukawa potential for $\hat{V}_{s}$, Eq. (1)], $\Phi_{n}^{\text {min,l }}$ will be cuspless. As the long-range electron-electron interaction is present in $\left\langle\Phi_{n}^{\min , l}\left|\hat{T}+\hat{V}_{l}\right| \Phi_{n}^{\min , l}\right\rangle$, only the shortrange parts of $U[n]$,

$$
U_{s}[n]=\frac{1}{2} \int n\left(\mathbf{r}_{1}\right) n\left(\mathbf{r}_{2}\right) v_{s}\left(\mathbf{r}_{1}, \mathbf{r}_{2}\right) d^{3} r_{1} d^{3} r_{2},
$$

and that of $\left\langle\Phi_{n}^{\min }\left|\hat{V}_{e e}\right| \Phi_{n}^{\min }\right\rangle$, namely, $\left\langle\Phi_{n}^{\min }\left|\hat{V}_{s}\right| \Phi_{n}^{\min }\right\rangle$, have to be subtracted in the expressions for $E_{s, x c}[n]$ and $E_{s, c}[n]$, respectively.

The ground-state energy, $E_{g s}$, can be obtained as for the full interaction density functionals by searching for the optimal wave function:

$$
\begin{aligned}
& E_{g s}=\min _{n}(\left.F[n]+\int n v_{e x t}\right) \\
&=\min _{\Phi}\left(\left\langle\Phi\left|\hat{T}+\hat{V}_{l}\right| \Phi\right\rangle+\int n(\Phi) v_{e x t}\right. \\
&\left.+U_{s}[n(\Phi)]+E_{s, x c}[n(\Phi)]\right) \\
&=\min _{\Phi}\left(\left\langle\Phi\left|\hat{T}+\hat{V}_{l}\right| \Phi\right\rangle+\int n(\Phi) v_{e x t}\right. \\
&\left.\quad+\left\langle\Phi_{n}^{\min }\left|\hat{V}_{s}\right| \Phi_{n}^{\min }\right\rangle+E_{s, c}[n(\Phi)]\right),
\end{aligned}
$$

where $n(\Phi)$ means that the density is determined via the wave function $\Phi$ and $v_{e x t}$ is the external potential. $\Phi_{n}^{\min }$ should be determined according to the definition above by using the standard Kohn-Sham equations [2].

It is possible to use the coupling-constant integration (adiabatic connection, see, e.g., [16]) in order to obtain more insight into the functionals $E_{s, x c}[n]$ and $E_{s, c}[n]$. The formulas are obtained with the Hamiltonian

$$
\hat{H}(g)=\hat{T}+\hat{V}_{l}+g \hat{V}_{s}+\hat{V}(g),
$$

where $\hat{V}(g)$ is a one-particle operator that contains the external potential and guarantees the conservation of $n$ during the process of changing the parameter $g(0 \leq g \leq 1)$. Formulas similar to those for $E_{x c}[n]$ and $E_{c}[n]$ can be then easily obtained. For example:

$$
\begin{aligned}
E_{s, x c}[n]=\frac{1}{2} \int_{0}^{1} d g \int d^{3} r_{1} d^{3} r_{2}\left[P_{2}\left(\mathbf{r}_{1}, \mathbf{r}_{2} ; g\right)\right. \\
\left.-n\left(\mathbf{r}_{1}\right) n\left(\mathbf{r}_{2}\right)\right] v_{s}\left(\mathbf{r}_{1}, \mathbf{r}_{2}\right) .
\end{aligned}
$$


$P_{2}\left(\mathbf{r}_{1}, \mathbf{r}_{2} ; g\right)$ is the second-order density matrix $[17,18]$.

The choice of $\hat{V}_{s}$ allows a continuous switch between pure density functional and configuration interaction calculations. For that given in Eq. (1), this amounts to changing $\mu$ from zero to infinity.

As for $E_{x c}[n]$ and $E_{c}[n]$, the short-range functionals can be obtained in an approximate form, the simplest one being the local one:

$$
E_{i}[n] \approx \int d^{3} r n(r) \epsilon_{i}(n(r)),
$$

where $i$ stands for any of $x c, c, s, x c$, or $s, c . \epsilon_{i}(n)$ is a function depending on the density alone. A convenient way to determine the $\epsilon_{i}$ is by using homogeneous electron gas calculations. Some details are given in the Appendix.

The local approximation with the electron gas fit has been tested for a few two-electron systems (the He series and the Be series; pseudopotentials have been used for the latter).

\section{Computational Details and Results}

The calculations were performed using large Gaussian-type $(s, p, d)$ basis sets [19] for the $\mathrm{He}$ series (He to $\mathrm{C}^{4+}$ ) and the Be series (Be to $\mathrm{O}^{4+}$ ). The number of variational parameters was reduced by contracting the Gaussians to the first $3 s, 2 p, 1 d$ natural orbitals (as in [20]). The semilocal pseudopotentials for the Be series were taken from $[21,22]$.

For two-electron systems, $\Phi_{n}^{\text {min }}$ is easily obtained from the densities; its spatial part is simply $(1 / 2)\left[n\left(\mathbf{r}_{1}\right) n\left(\mathbf{r}_{2}\right)\right]^{1 / 2}$ (see, e.g., [23]). $\hat{V}_{s}$ was chosen as in Eq. (1), the parameter $\mu$ being varied between 0 and 24 bohr $^{-1}$.

Both the calculations with $E_{s, x c}$ and with $E_{s, c}$ were done self-consistently. At least for the systems studied, the error is small when using $n$ and $\Phi$ from the $E_{s . \ldots}$ calculation for obtaining the energy with the density functional for $E_{s, c}$ alone.

The difference between the value calculated in the local density approximation for $\hat{V}_{s}$ and the "exact" values ([24] for the He series, and the full configuration interaction results for the Be series) is presented in Figures 1 and 2. There is a systematic shift of the curves with the change in the nuclear charge. This is related to the fact that $1 / \mu$ corresponds to an effective interaction radius, which,
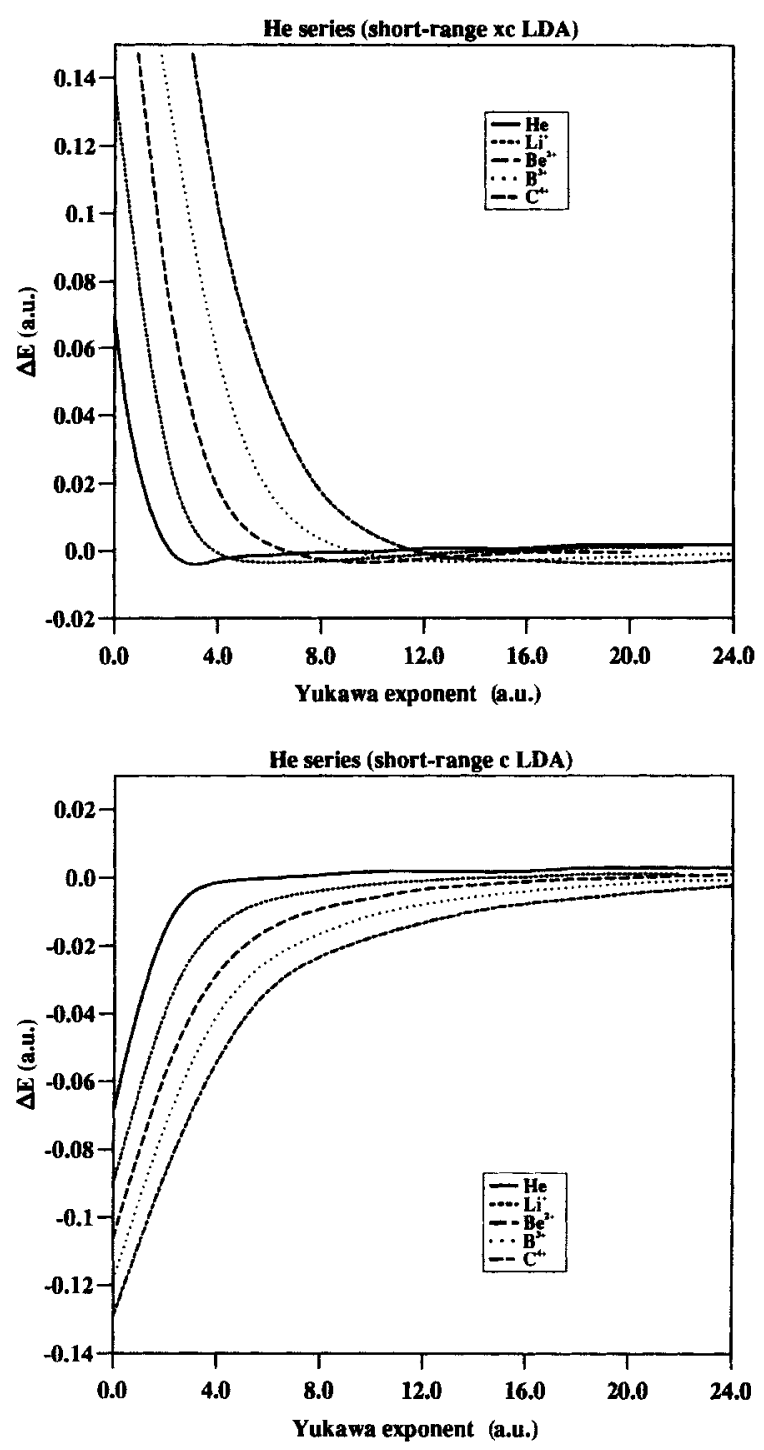

FIGURE 1. He series. Difference between the calculated (local density approximation for Yukawa short-range electron-electron interaction) and "exact" total energies with density functionals for exchange and correlation (top) or for correlation only (bottom); as functions of the Yukawa exponential factor; in atomic units.

of course, becomes smaller within an isoelectronic series with increasing $Z$. The values for $\mu=0$ correspond to usual local density calculations. An increase of the Yukawa exponent $\mu$ in the vicinity of zero always leads to a decrease of the magnitude of the error. After a certain value of $\mu, \mu_{0}$, the errors remain small (of the order of $10^{-3}$ Hartree). $\mu_{0}$ seems to be smaller with exchange-correlation density functionals than for those for correlation only, 

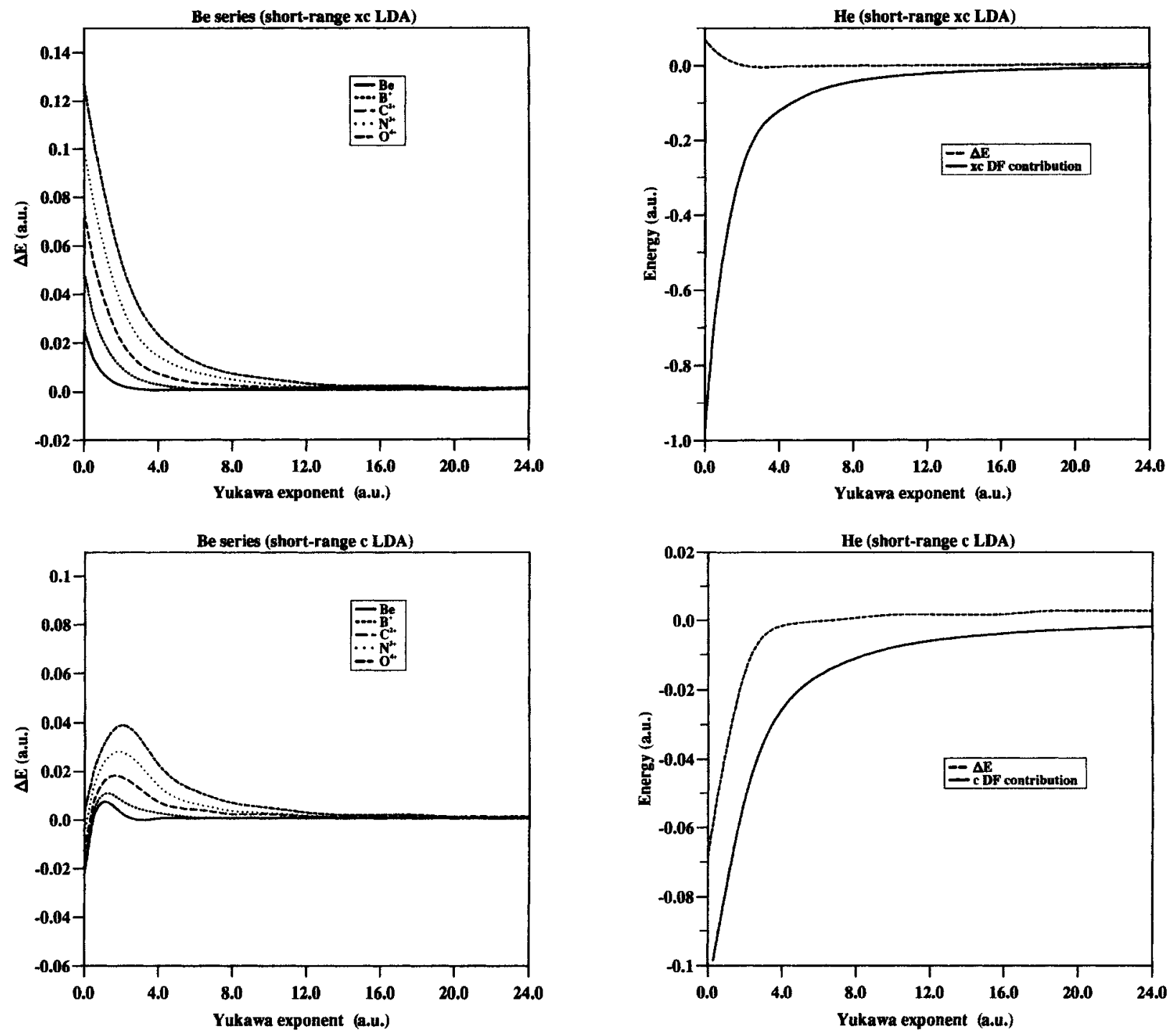

FIGURE 2. Be series. Difference between the calculated (local density approximation for Yukawa short-range electron-electron interaction) and "exact" total energies with density functionals for exchange and correlation (top) or for correlation only (bottom); as functions of the Yukawa exponential factor; in atomic units.

even when the error at $\mu=0$ (usual local density approximation) shows the opposite trend. This might be related to the damping of the long-range effects in the homogeneous electron gas (see, e.g., [25]) when correlation and exchange are treated together. Another explanation might be the difference in the sizes of the exchange and correlation holes (the latter being smaller needs a larger $\mu$ ).

For $\mu \rightarrow \infty$, the configuration interaction results are obtained. The question arises whether the local 


\section{Conclusions}

The use of short-range density functionals leads to encouraging results in two-electron systems, even when using the local density approximation. One might use this in order to have a controlled improvement of the calculated energy. In practice, one might either rely on experience for choosing the optimal $\mu$ or examine the stability of the obtained result with respect to changes in $\mu$.

Further calculations have to study systematically the convergence in the wave-function expansion in terms of Slater determinants in connection with the present schemes. It will be also useful to explore other definitions of $\hat{V}_{s}$. For calculations with Gaussian basis sets, the form $\exp \left(-\mu r_{12}^{2}\right) / r_{12}$ might be preferable. Further consideration should be given to more sophisticated forms of the density functionals (depending on the gradient, spin-polarization, or second-order density matrix).

\section{ACKNOWLEDGMENTS}

We are grateful to Prof. E. Krotscheck (Texas A\&M University) for the FHNC homogeneous electron gas program and to Prof. H.-J. Werner (Universität Stuttgart, Germany) for the configuration interaction program Molpro [26]. We would like to thank the IDRIS (Orsay, France) for the allocation of computer time.

\section{Appendix: Homogeneous Electron Gas Calculations for the Local Approximations of Short-range Density Functionals}

The homogeneous electron gas is very convenient for obtaining the dependence of $\epsilon_{1}$ (energy per particle) on $n$ needed for Eq. (2). To obtain the short-range $\epsilon_{i}$ with $\hat{V}_{s}$ given by Eq. (1), the Fourier transform of the $\hat{V}_{s}$ is needed, which is $4 \pi /\left(k^{2}+\right.$

TABLE I

Ratio between the short-range and total correlation energies in the Fermi-hypernetted chain approximation $\left[r_{s}=[3 /(4 \pi n)]^{1 / 3}, \mu\right.$ is the Yukawa exponent in Eq. (1), in atomic units].

\begin{tabular}{|c|c|c|c|c|c|c|c|}
\hline \multirow[b]{2}{*}{$\mu$} & \multicolumn{7}{|c|}{$r_{s}$} \\
\hline & 0.2 & 0.5 & 1.0 & 2.0 & 3.0 & 4.0 & 5.0 \\
\hline 0.2 & 0.999 & 0.991 & 0.962 & 0.898 & 0.835 & 0.769 & 0.705 \\
\hline 0.5 & 0.991 & 0.935 & 0.849 & 0.697 & 0.564 & 0.453 & 0.362 \\
\hline 1.0 & 0.948 & 0.830 & 0.679 & 0.455 & 0.306 & 0.209 & 0.149 \\
\hline 2.0 & 0.846 & 0.655 & 0.446 & 0.222 & 0.121 & 0.071 & 0.046 \\
\hline 3.0 & 0.757 & 0.525 & 0.309 & 0.128 & 0.063 & 0.035 & 0.023 \\
\hline 4.0 & 0.681 & 0.428 & 0.223 & 0.081 & 0.038 & 0.020 & 0.013 \\
\hline 5.0 & 0.616 & 0.354 & 0.168 & 0.056 & 0.025 & 0.013 & 0.008 \\
\hline 6.0 & 0.560 & 0.296 & 0.130 & 0.040 & 0.018 & 0.009 & 0.006 \\
\hline 7.0 & 0.511 & 0.251 & 0.103 & 0.031 & 0.014 & 0.007 & 0.004 \\
\hline 8.0 & 0.468 & 0.216 & 0.085 & 0.025 & 0.011 & 0.005 & 0.004 \\
\hline 9.0 & 0.430 & 0.187 & 0.070 & 0.020 & 0.008 & 0.004 & 0.002 \\
\hline 10.0 & 0.396 & 0.163 & 0.059 & 0.016 & 0.006 & 0.004 & 0.002 \\
\hline 11.0 & 0.367 & 0.144 & 0.050 & 0.013 & 0.005 & 0.002 & 0.002 \\
\hline 12.0 & 0.340 & 0.127 & 0.043 & 0.011 & 0.005 & 0.002 & 0.002 \\
\hline 13.0 & 0.315 & 0.113 & 0.038 & 0.010 & 0.003 & 0.002 & 0.002 \\
\hline 14.0 & 0.294 & 0.102 & 0.034 & 0.009 & 0.003 & 0.002 & 0.002 \\
\hline 15.0 & 0.274 & 0.092 & 0.029 & 0.007 & 0.003 & 0.001 & 0.001 \\
\hline 16.0 & 0.256 & 0.083 & 0.027 & 0.006 & 0.003 & 0.001 & 0.001 \\
\hline 17.0 & 0.240 & 0.075 & 0.024 & 0.006 & 0.002 & 0.001 & 0.001 \\
\hline 18.0 & 0.225 & 0.069 & 0.021 & 0.005 & 0.002 & 0.001 & 0.001 \\
\hline 19.0 & 0.211 & 0.063 & 0.019 & 0.005 & 0.002 & 0.000 & 0.000 \\
\hline 20.0 & 0.199 & 0.058 & 0.018 & 0.005 & 0.002 & 0.000 & 0.000 \\
\hline 21.0 & 0.188 & 0.054 & 0.016 & 0.004 & 0.002 & 0.000 & 0.000 \\
\hline 22.0 & 0.177 & 0.050 & 0.015 & 0.004 & 0.002 & 0.000 & 0.000 \\
\hline 23.0 & 0.168 & 0.046 & 0.014 & 0.004 & 0.002 & 0.000 & 0.000 \\
\hline 24.0 & 0.159 & 0.043 & 0.012 & 0.002 & 0.002 & 0.000 & 0.000 \\
\hline 25.0 & 0.151 & 0.040 & 0.011 & 0.002 & 0.000 & 0.000 & 0.000 \\
\hline
\end{tabular}


$\mu^{2}$ ) instead of $4 \pi / k^{2}$ for $\hat{V}_{e e}$. For exchange, $\epsilon_{i}$ can be easily obtained in analytical form by replacing $4 \pi / k^{2}$ in the usual formulas (given, e.g., in [27]):

$$
\begin{aligned}
\epsilon_{s, x}=-\frac{2}{\pi} k_{F} & \left(\frac{3}{8}-a \tan ^{-1} \frac{1}{a}\right. \\
& \left.+\frac{3+a^{2}}{4} a^{2} \ln \frac{1+a^{2}}{a^{2}}-\frac{a^{2}}{4}\right),
\end{aligned}
$$

where $a=\mu /\left(2 k_{F}\right)$ and $k_{F}=(3 \pi n)^{1 / 3}$.

For the short-range correlation $\left(\epsilon_{s, c}\right)$, more elaborate calculations are needed. For this article, the Fermi-hypernetted-chain (FHNC//0 approximation) program of Krotscheck was changed only by modifying the electron-electron interaction [28]. For the total correlation energy per particle (obtained with $\hat{V}_{s}=\hat{V}_{e e}$ ), the values obtained are typically 0.005 to 0.010 Hartree lower than those obtained with the Vosko-Wilk-Nusari (VWN) formula [29]. To have a comparison with standard local-density calculations, the FHNC $\epsilon_{s, c}$ were scaled with the factors given by the ratio between the VWN $\epsilon_{c}$ and the FHNC//0 one. The ratio between $\epsilon_{s, c} / \epsilon_{c}$ in the $\mathrm{FHNC} / / 0$ approximation are given in Table I. The atomic program interpolated between these values [30]. The integrations were performed numerically, although analytical formulas can be derived. This was done in order to have a greater flexibility in the choice of $\hat{V}_{s}$ and $\hat{V}_{l}$.

\section{References}

1. P. Hohenberg and W. Kohn, Phys. Rev. B 136, 864 (1964).

2. W. Kohn and L. Sham, Phys. Rev. A 140, 1133 (1965).

3. W. Kutzelnigg and J. D. Morgan III, J. Chem. Phys. 97, 4484 (1992); lbid. 97, 8821 (1992).

4. B. O. Roos, Adv. Chem. Phys. 69, 399 (1987).

5. G.C. Lie and E. Clementi, J. Chem. Phys. 60, 1274 (1974).
6. T. Ziegler, A. Rauk, and E.J. Baerends, Theor. Chim. Acta 43, 261 (1977).

7. R. Colle and O. Salvetti, Theor. Chim. Acta 53, 55 (1979).

8. H. Stoll and A. Savin, in Density Functional Methods in Physics, R. M. Dreizler and J. da Providencia, Eds. (Plenum, New York, 1985), p. 177.

9. L. Fritsche, Phys. Rev. B 33, 3976, 1986.

10. B. O. Roos, M. Szulkin, and M. Jaszuński, Theor. Chim. Acta 71, 375 (1987).

11. F. Moscardo and E. San-Fabian, Phys. Rev. A 44, 1549 (1991).

12. A. Savin, in Density Functional Methods in Chemistry, J.K. Labanowski and J.W. Andzelm, Eds. (Springer, New York, 1991), p. 213.

13. A. Görling and M. Levy, Phys. Rev. B 47, 13105 (1993).

14. M. Levy, Proc. Natl. Acad. Sci. U.S.A. 76, 6062 (1979).

15. J.K. Percus Int. J. Quantum Chem. 13, 89 (1978).

16. O. Gunnarsson and B.I. Lundqvist, Phys. Rev. B 13, 4274 (1976).

17. P.-O. Löwdin, Phys. Rev. 97, 1474 (1955).

18. R. McWeeny, Proc. R. Soc. Lond. A 232, 114 (1955).

19. F. B. van Duijneveldt, IBM Res. Rep. 945 (1971).

20. J. Almolöf and P. R. Taylor, J. Chem. Phys. 86, 4070 (1987).

21. Igel-Mann, H. Stoll, and H. Preuss, Mol. Phys. 65, 1321 (1988).

22. P. Fuentealba, L. v. Szentpaly, $H$. Preuss, and H. Stoll, J. Phys. B 18, 1287 (1985).

23. Y. Tal and R.W.F. Bader, Int. J. Quantum Chem. S12, 153 (1978).

24. K. Frankowski and C. L. Pekeris, Phys. Rev. 146, 46 (1966).

25. D.C. Langreth and J. P. Perdew, Solid State Comm. 17, 1425 (1975); Phys. Rev. B 15, 2884 (1977).

26. P. J. Knowles and H.-J. Werner, Chem. Phys. Lett. 115, 259 (1985); Ibid. 145, 514 (1988); H.-J. Werner and P. J. Knowles, J. Chem. Phys. 82, 5033 (1985); Ibid. 89, 5803 (1988).

27. A.L. Fetter and J.D. Walecka, Quantum Theory of ManyParticle Systems (McGraw Hill, New York, 1971), p. 28.

28. E. Krotscheck, W. Kohn, and B.-X. Qian, Phys. Rev. B 32, 5693 (1985).

29. S. H. Vosko, L. Wilk, and M. Nusair, Can. J. Phys. 58, 1200 (1980).

30. W.H. Press, B.P. Flanery, S. A. Teukowsky, and W. T. Vetterling, Numerical Recipes (Cambridge University Press, Cambridge, 1986), p. 85. 